\title{
Monitor Scale
}

National Cancer Institute

\section{Source}

National Cancer Institute. Monitor Scale. NCI Thesaurus. Code C121472.

A rating scale included in the Behavior Rating Inventory of Executive Function that assesses work-checking habits and interpersonal awareness. The task monitoring portion of the scale captures whether a child assesses his or her own performance during or shortly after finishing a task to ensure accuracy or appropriate attainment of a goal. The self-monitoring portion of the scale evaluates whether a child keeps track of the effect that his or her behavior has on others. 